\title{
Adaptive Color Visualization for Dichromats using a Customized Hierarchical Palette
}

\author{
Carlos Eduardo Rodríguez-Pardo and Gaurav Sharma \\ ECE Dept. Univ. of Rochester, Rochester, NY, USA
}

\begin{abstract}
We propose a user-centric methodology for displaying digital color documents, that optimizes color representations in an observer specific and adaptive fashion. We apply our framework to situations involving viewers with common dichromatic color vision deficiencies, who face challenges in perceiving information presented in color images and graphics designed for color normal individuals. For situations involving qualitative data visualization, we present a computationally efficient solution that combines a customized observer-specific hierarchical palette with "display time" selection of the number of colors to generate renderings with colors that are easily discriminated by the intended viewer. The palette design is accomplished via a clustering algorithm, that arranges colors in a hierarchical tree based on their perceived differences for the intended viewer. A desired number of highly discriminable colors are readily obtained from the hierarchical palette via a simple truncation. As an illustration, we demonstrate the application of the methodology to Ishihara style images.
\end{abstract}

Keywords: Color palette design, dichromacy, customized design, hierarchical clustering

\section{INTRODUCTION}

It is known that nearly $8 \%$ of the male population present some kind of color deficiency. A significant proportion of color vision problems are the consequence of the absence of one particular type of photoreceptor in the eye, a condition known as dichromacy. Dichromats are prone to loose some information when presented a color document designed for a color normal observer, which is a common situation when exposed to the screen of a computer, TV or most of the mobile and media player devices. Some color visualization methodologies, aware of this impairment, propose guidelines for the selection of color palettes, which is particularly useful for printed documents like maps and emergency signs. ${ }^{5}$ There also exist software programs that simulate colorblind vision, and help designers to choose suitable sets of colors. ${ }^{1,3,4}$ However, within a single representation, it is impossible to satisfy every different requirement in all situations.

We are interested in making the information conveyed by color more accessible to all viewers. Rather than find a single solution that incorporates all possible restrictions, our approach, explores the alternative of a usercentered design. Although such approaches were computationally intractable a few years ago, the rapid advances in computational infrastructure offer an opportunity for exploring personalized solutions. In fact, a number of the major challenges in information visualization nowadays can be formulated from a user-centered perspective. ${ }^{7}$

The methodology we propose incorporates the characteristics of different observers in a system that selects for them a color palette that best represents the information conveyed in a digital document when it is accessed. There are two principal aspects that determines the success of this kind of systems. First, how effectively the user is characterized in order to generate the information to render the document, and second, the way the information is organized such that it is ready whenever the user needs it.

To address the problem, we first introduce in Section 2 some notions of color representation that will be use to build the profiles of the different users. In Section 3 we describe our methodology in more detail. The palette selection algorithm based on hierarchical clustering is described in Section 4. In Section 5 we present some results, and we conclude and describe future alternatives in Section 6.

Further author information:

C.R.: E-mail: pardo@ece.rochester.edu, Telephone: 15852758122

G.S.: E-mail: gaurav.sharma@rochester.edu

Color Imaging XVI: Displaying, Processing, Hardcopy, and Applications, edited by Reiner Eschbach, Gabriel G. Marcu, Alessandro Rizzi, Proc. of SPIE-IS\&T Electronic Imaging, SPIE Vol. 7866 786603 • (c) 2011 SPIE-IS\&T · CCC code: 0277-786X/11/\$18 doi: 10.1117/12.872601 


\section{COLOR REPRESENTATION FOR TRICHROMATS AND DICHROMATS}

\subsection{Vector Space Description for Color Vision}

The eye of a color-normal observer has, embedded in the retina, three types of photoreceptors that govern color sensation. These photoreceptors are known as the $L, M$ and $S$ cones and are sensitive to the long, medium and small wavelength regions of the visual spectrum, respectively ${ }^{15}$ Therefore the color sensation produced by a light incident on the eye depends on its power spectral distribution, denoted in this paper by $f(\lambda)$, where $\lambda$ represents the wavelength. The responses each of the three cones are modeled as

$$
\begin{aligned}
c_{l} & =\int s_{l}(\lambda) f(\lambda) d \lambda, \\
c_{m} & =\int s_{m}(\lambda) f(\lambda) d \lambda, \\
c_{s} & =\int s_{s}(\lambda) f(\lambda) d \lambda,
\end{aligned}
$$

where here $s_{i}(\lambda)$, with $i=l, m, s$ are the sensitivity functions for the $i^{t h}$ type of cone. The integration is computed over the all wavelengths, but it can be limited to the interval [360 $\mathrm{nm}, 830 \mathrm{~nm}]$, that approximately contains the visible spectrum or, equivalently, the interval of wavelengths where at least one of the cone sensitivities is nonzero.

For computation purposes, the spectra of the light and the cone sensitivities can be represented by a vectors of $N$ samples obtained in the visible spectrum, denoted by $\mathbf{f}, \mathbf{s}_{i}$, respectively. Usually one sample every $10 \mathrm{~nm}$ provides enough accuracy in most of the cases. ${ }^{20}$ Following closely the notation in, ${ }^{17}$ if we define $\mathbf{S}=\left[\mathbf{s}_{l}, \mathbf{s}_{m}, \mathbf{s}_{s}\right]$ as the $N \times 3$ matrix of sensitivities and the $3 \times 1$ cone responses vector $\mathbf{c}=\left[c_{l}, c_{m}, c_{s}\right]^{T}$, then

$$
\mathbf{c}=\mathbf{S}^{T} \mathbf{f} .
$$

This expression suggests that the cone responses are the inner-products of the cone sensitivities and the incident spectrum $f$. Hence, the cone responses can be used to determine the projection of the spectrum onto the space spanned by the three sensitivity functions, $\mathbf{s}_{i}$. This space is called the trichromatic human visual subspace, or simply the HVSS.

In normal observers the sensitivities are linear independent, so the HVSS is a 3 -dimension subspace of the $N$-dimensional spectral space. This implies that there are multiple spectra that are projected to the same point in the HVSS, producing the same color sensation making them indistinguishable for the human eye. In that case we say that the spectra match in color, in fact, two spectra $\mathbf{f}, \mathbf{g}$ match in color if and only if

$$
\mathbf{S}^{T} \mathbf{f}=\mathbf{S}^{T} \mathbf{g} .
$$

\subsection{Dichromacy}

Color vision deficiency in human vision is often caused by a loss of sensing ability in one or more cone types. People with only two cone responses are called dichromats, and they are divided in three groups: protanopes, deuteranopes and tritanopes, depending on whether they are missing the $L, M$, or $S$ cones, respectively. Monochromats are individuals with only one type of cone, and this form of color vision impairment is the most extreme and also the most rare. ${ }^{15}$

The sensation of color perceived by a dichromatic individual will be reduced to two dimensions as opposed to the normal three dimensions for a trichromatic observer. For example, in the particular case of a protanope as an example, the color sensation will depend only on the $c_{m}, c_{s}$ cone responses. That is, if we define the matrix $\mathbf{M}_{p}=\left[\mathbf{e}_{2}, \mathbf{e}_{3}\right]$, where $\left\{\mathbf{e}_{i}\right\}_{i=1}^{3}$ is the standard basis for $\mathbf{R}^{3}$, then the cone responses for protanopes will be,

$$
\left[c_{m}, c_{s}\right]^{T}=\mathbf{S}_{p}^{T} \mathbf{f},
$$

where $\mathbf{S}_{p}=\mathbf{S M}_{p}$. This means that $f$ is projected onto the space spanned by columns of $\mathbf{S}_{p}$, which define the the protanope human visual sub-space $\mathrm{HVSS}_{p}$. In a similar fashion the deuteranope and tritanope human visual subspaces, $\mathrm{HVSS}_{d}$ and $\mathrm{HVSS}_{t}$ can be defined. Therefore we can consider that there are three dichromatic visual subspaces, and as seen from (4), each of these spaces are 2-dimensional subspaces of the original trichromatic HVSS. 
This implies the existence of spectra stimuli that color match for a dichromatic observer, but are differentiated by trichromatic individuals. For instance, if $\mathbf{c}$ is the trichromatic cone response for a spectra $\mathbf{f}$, the protanope will confuse or perceive as the same, all spectra $g$ which cone response lies on the confusion line defined by parametric equation,

$$
\mathbf{r}_{\mathbf{c}}(t)=t \mathbf{e}_{1}+\mathbf{c}, \quad t \in \mathbf{R},
$$

which is a line parallel to the vector $\mathbf{e}_{1}$ that passes through $\mathbf{c}$. Confusion lines characterize each of the types of dichromacy and help to determine sets of colors that are confused by color deficient individuals.

The tristimulus space defined by the cone responses represents colors in the first stage in the color vision process. Perceptual color spaces, such as CIELAB, provide a representation that better models the appearance of colors. These perceptual color spaces are defined by nonlinear transformations of the cone tristimulus space that in addition to providing perceptual appearance correlates also have the characteristic that the Euclidean distance between color representations in these spaces approximates the magnitude of perceived difference. ${ }^{16} \mathrm{~A}$ color in the CIELAB space is described in terms of three variables: $L^{*}$ that gives the lightness of the color and $a^{*}, b^{*}$ that contain the chromaticity information.

With perceptual spaces we can appreciate, in particular, the differences in perception between dichromats and color-normal observers. Based on, ${ }^{6}$ we simulate the perception of dichromats. Specifically, based on studies of unilateral dichromats, i.e. individuals who are dichromatic in one eye and have normal trichromatic vision in the other eye, for each confusion line for a dichromat, the method proposed in $^{6}$ allows us to identify a unique point on the confusion line for which the perceptions of dichromatic and trichromatic observers match. This allows us to generate, for any given color stimulus, a rendition that simulates, for a trichromatic observer, the appearance of the stimulus for a given dichromat. As an example consider a set of colors that only vary in hue, (Lightness L and the the Chroma $C=\sqrt{a^{* 2}+b^{* 2}}$ are constant), forming a hue circle as shown in Fig. 1. It can appreciated the difference in perception between the trichromats and the different types of dichromatic observers. The perceived colors are grouped on an ellipsoid-shaped way, changing lightness and hue. The solid line represents the confusion line for the color in the center of the original circle. Figure 1 also shows the projection on the $a^{*}, b^{*}$ plane, where it can be appreciated that hue for the perceived colors collapses on to a line.

\section{CUSTOMIZED VISUALIZATION FOR DICHROMATS}

A customized visualization approach exploits individual user characteristics to create a representation that transmit as best as possible the information contained in an image. There are many possible users as well as many possible information to convey. We consider user profiles that characterize users in terms of their color vision as trichromats, protanopes, deuteranopes and tritanopes. Although the methodology we present can be applied in a most general scenario, the results we show in this work are focused on the case of documents containing a single image where qualitative differences between different categories are to be highlighted by the use of different colors. Bar graphs and pie charts represent specific examples of these types of image representations used for visualizing scientific data.

\subsection{Color Information Visualization}

Suitable colors for image representations can be chosen by considering different aspects regarding the image itself and the observer. On the one hand, it has been shown that color distance and color category are features of color that can influence the rapid identification of colors for a given observer. ${ }^{9}$ Complementing these ideas, some other works propose guidelines for palette selection for scientific data based on the purpose of the information to convey. For example, qualitative palettes, where the same perceptual importance is given to all colors, is preferred when categorical data is transmitted, like bar graphs, pie charts, etc. Sequential palettes, where colors with lightness differences are assigned, are suggested for numerical variables whose value ranges in an interval. For classification maps, diverging palettes, that mix the qualitative and sequential palette instructions, are considered as the best option. ${ }^{5}$ On the other hand, images are perceived different for observers with different characteristics, as the example of dichromats. Although it is possible to derive palettes that avoid some colors that present difficulty to color blind individuals, ${ }^{21}$ there is not clear suggestions of what colors are indeed the best choice for them. 

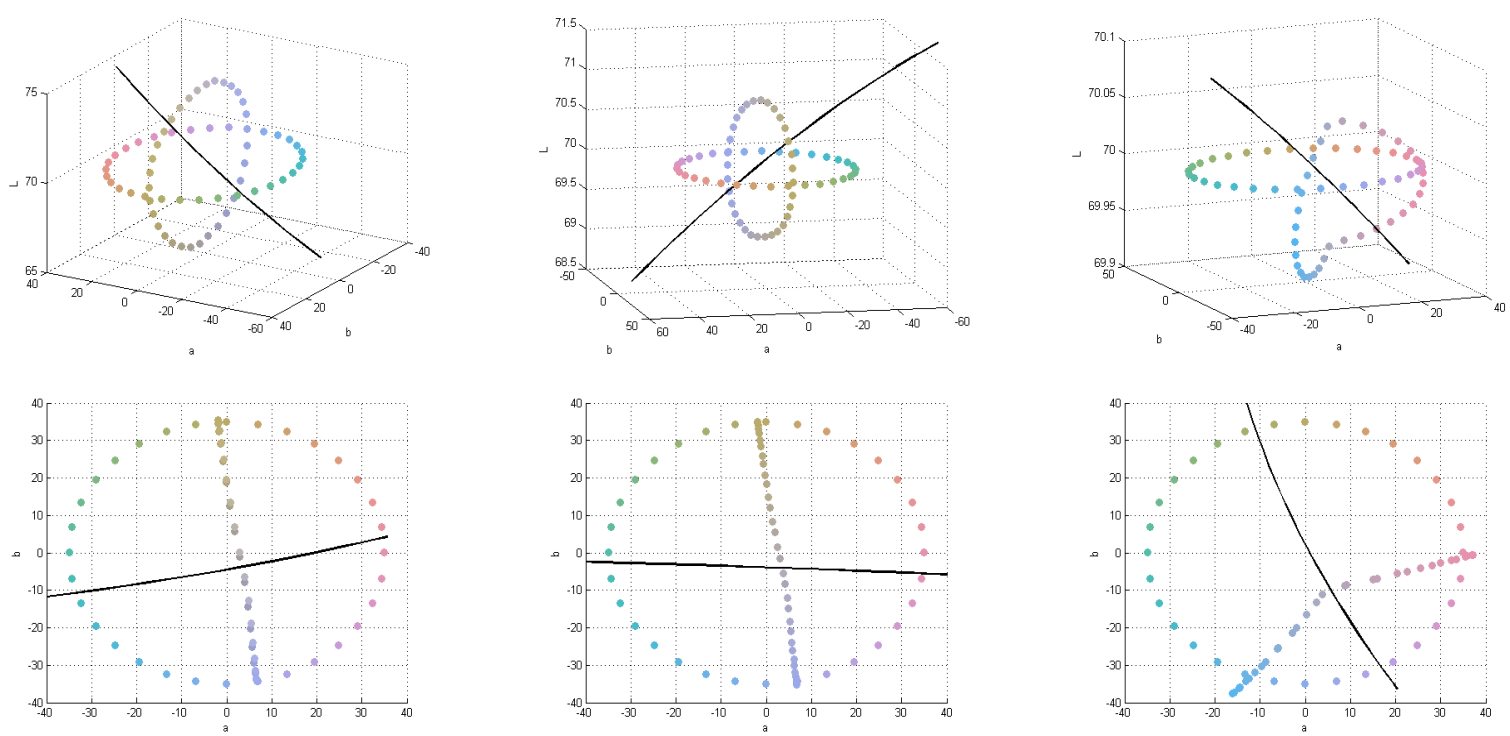

Figure 1. Visualization of the dichromat perception in CIELAB space. The figures in the top row show a set of colors located along a $\mathrm{L}^{*}=75$ and $\mathrm{C}=35$ hue circle (in each image) and the corresponding perceived colors for protanopes (left column), deuteranopes (center) and tritanopes (right column). The perceived colors for the dichromats lie on deformed ellipses. Through the center of these ellipses passes the confusion line for a neutral color of luminance $\mathrm{L}^{*}=75$. The bottom row shows the projections of the perceived colors for the dichromatic observers on the $\mathrm{a}^{*}, \mathrm{~b}^{*}$ plane. The hue for the perceived colors collapses onto a line for the dichromats. A confusion line for each case is also illustrated.

For those images that are not designed for the colorblind population, there is the alternative of using recolorization strategies. Some of this work can be found in. ${ }^{11,13,14,19}$ Most of these methods are oriented toward natural images, where modification in the lightness contrast is a common strategy for enhancing the content of images and make it more understandable for colorblind individuals. These techniques are document dependent and usually slow. ${ }^{10}$ These methods do not include criteria described above, and therefore are not necessarily optimal for the purpose of communicating data with scientific information.

\subsection{Methodology}

The objective is to choose based on the characteristics a given graph, the colors that best convey the information when the image is seen by a predefined user.

Our methodology for customized color visualization is summarized in Fig. 2 using a bar chart as an example graphic to be displayed (where a qualitative palette is appropriate). The process starts when a user, whose profile has been previously introduced in the system, selects the digital graphic to be displayed. Then, the number of colors required for the display are determined from the graphic This number can be determined either through an analysis of the graphic or more preferably is pre-determined at the time of design and embedded along with the graphic in the form of rendering hints. This information could be included, for instance, in augmented versions of ICC Profiles ${ }^{8}$ that already include other rendering hints for color normal observers. This step of obtaining features relevant for rendering can be extended to more general scenarios by considering additional image characteristics that help to render the graphic satisfactorily. In the next step, a palette selection for the graphic is performed to offer the user an adequate visualization. In our case, this can obtained by maximizing the distance between colors as perceived by the specific dichromatic user, so that the data can be easily discriminated. Finally, once the palette is selected a color mapping is applied to the document and then it is displayed to the user.

The rendering of each graphic must be generated adaptively for the user at the time the graphic is displayed, imposing speed constraints for the palette selection step. Given that the user is already known, different palette options that satisfy their needs can be created beforehand, such that when the algorithm runs, it selects one 


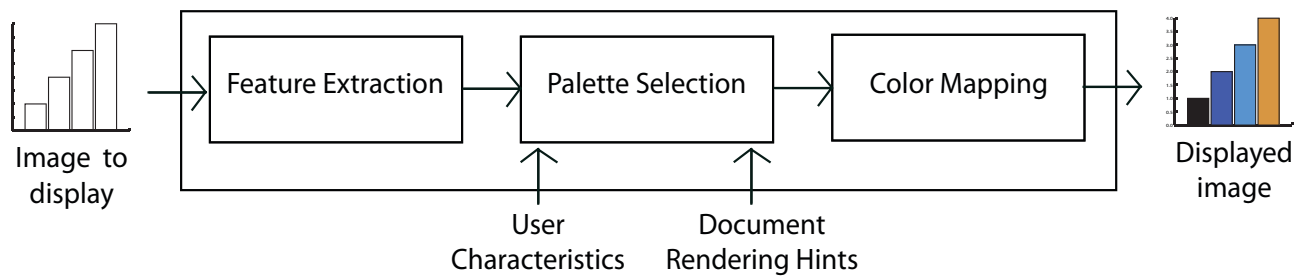

Figure 2. Diagram of blocks describing the methodology proposed. The palette selection is the core of the system, which depends on the profile of the user and the characteristics of the image.

of them depending only on the number of colors, reducing latency, which is an important factor for users. The reduced latency comes at the expense of slightly increased memory use, which is not likely to pose challenges in practice.

\section{HIERARCHICAL PALETTE DESIGN}

We propose to use a hierarchical clustering algorithm to determine colors to be displayed, according to the number of colors required in a graph, for a predetermined observer. The system is restricted to finite set of colors that can be used to color a graphic. The idea is to establish a correspondence between the number of clusters for this set, and the number of colors needed in a graphic. That is, the objective is to color the graph by using one representative per cluster.

An adequate selection of colors maximizes the perceptual difference between the colors in the graph. For this objective, colors are described in the CIELAB space. Although the perception of trichromats rely on a three dimensional space, as seen in Section 2.2, a two dimensional subspace contains the colors perceived by dichromats. A simulation of the perception of colorblind individuals can be found in, ${ }^{6}$ which we use to represent in CIELAB the colors they perceive, which allows meaningful computations of perceptual distances for dichromats.

In an agglomerative, or bottom-up hierarchical clustering approach, the initial set of $N$ colors are grouping starting from the disjoint clustering, (i.e, every color represents a group) for a total of $N$ clusters. In the next step, $N-1$ groups are forming by merging two of groups in the previous level based on the inter-cluster distances. This is repeated, until all colors are merged into one group. ${ }^{12}$ The merging relies on the (user specific) dichromatic perceptual differences. At each stage, the colors judged to be perceptually the closest for the dichromatic observer are merged together. The hierarchical clustering can be described in a dendrogram graph as shown in Fig. 3. If the clustering is applied using the perceived colors by a predetermined user, then in the level $i, 1 \leq i \leq N$, of the dendrogram we find $i$ groups that are perceptually different one from each other. In that sense one representative per each cluster in the level $i$, form a palette selection when $i$ colors are required. At any level of the clustering the colors represent a set with given number of colors that best preserve distinguishability for the observer, which is the desired characteristic for the choice of colors for rendering the graphic for the observer.

\section{RESULTS}

\subsection{Designing a palette}

Consider first the simple case where the system has access to only 16 colors with constant chroma and luminance, $L^{*}=75, C=35$, respectively, and only the hue varies in a uniform way. This set, which is well-suited for highlighting qualitative differences, will be denoted as the uniform color set, and as a matter of notation, the palette of $N$ colors obtained with our algorithm from this set, will be denoted as the $N$-uniform palette

Consider the case where only four colors are needed to plot a graph. Once the hierarchical clustering is done, the dendrogram is cut on level four. The representatives of the clusters are chosen to to maximize the distance between the representative and the mean distance to the other clusters, although other criteria can be applied.

Figure 4 shows the results for applying the hierarchical clustering for the uniform color set. It can be appreciated the palette selection is based on the characteristic of the specific user, as approximated by the corresponding color representation in the CIELAB space. In this particular case, the original color set selected 


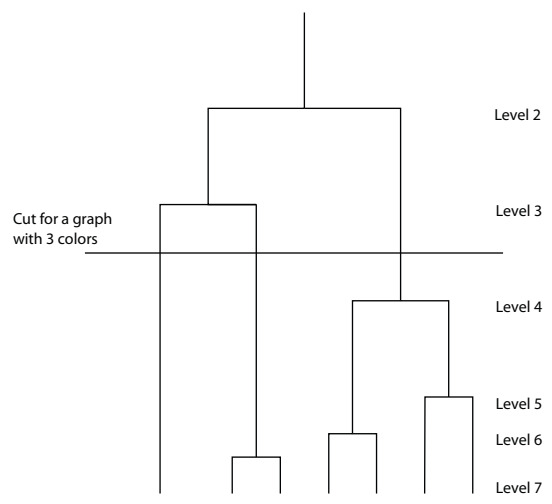

Figure 3. Dendrogram of a hierarchical clustering. Each node in the graph represent a cluster of colors. There is correspondence of the number of colors to use and the level in the dendrogram. In this case, an image with 3 colors use representatives of the clusters in the level 3

only varies the hue uniformly, but the perceived colors are very close, and in some cases only the lightness difference is relevant. Although the algorithm selects colors that are perceptually different, for graphs that require a large number of colors, eliminating all potential confusion is impossible. In fact, it was noted that for this case, graphs that require more than six colors will eventually use colors where differentiation is hard to perceive for some of the color deficient individuals. Alternate strategies for the selection of the initial palette would also be worth exploring.

\subsection{Rendering a Document}

To give an idea of the methodology proposed, consider a restriction to the uniform color set defined in Section 5.1. Our system has already run the hierarchical clustering, to determine all possible $N$-uniform palettes for the different users.

Consider a user that wants to visualize a document that contains a Ishihara style image as shown in Fig. 5, where it is also simulated how the image is perceived according to the observers. Note that as illustrated by the simulation, the text is not legible for a deuteranope. For the purpose of demonstration, suppose that the feature extraction block determined that the image can be displayed adequately by using only two colors that separate the text from the rest of the background image. Then, the palette selection block selects the 2-uniform palette for the user profile. For the mapping block, we determine the regions where the colors are applied by segmenting the image in two sets, based on the chroma content of the pixels. Figure 6 shows the result of this process, where the final displayed image as seen for the different users, can be appreciated.

\section{CONCLUSION}

For displaying color documents, the user-customized and document-adaptive approach proposed in this paper offers an attractive alternative for better communicating information to observers with differing color discrimination capabilities. Specifically, for users with dichromatic color vision deficiencies, we illustrate an application of the framework that allows computationally efficient customized and adaptive rendering of graphics through the use of suitably designed user-specific hierarchical palettes.

This work represents a first step in customized color information visualization. Although only a restricted application scenario is addressed in this paper, the framework presented here can be extended in several directions to address a much wider class of application scenarios. Two are of particular interest. First, within the domain of dichromatic color visualization, it is of interest to consider a broader class of imagery, including not only other types of graphics, but also pictorial images. Second, given variations between trichromatic "color normal" observers ${ }^{18}$ user customization of visualization is also of interest for this much larger segment of the population. 

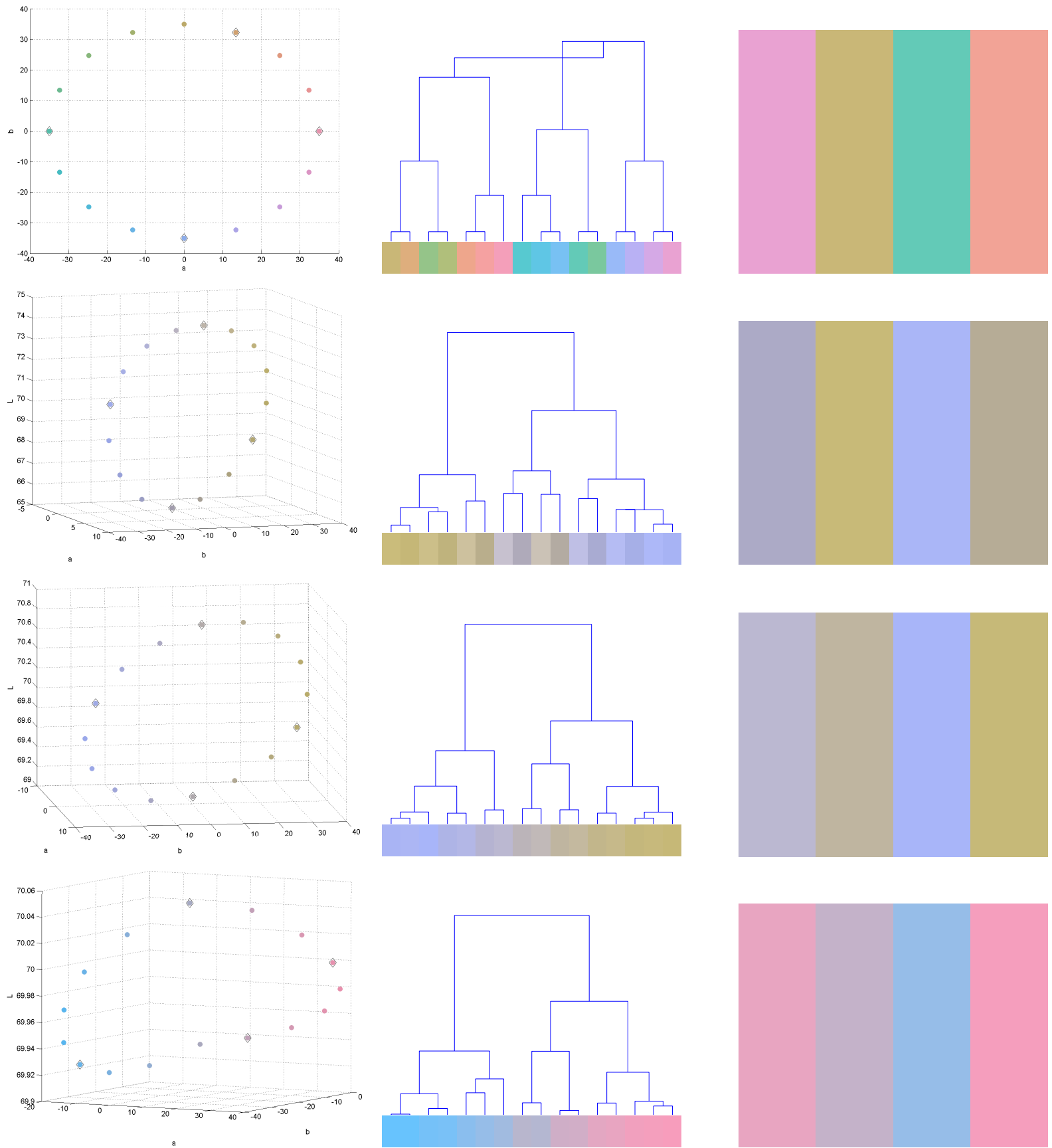

Figure 4. From top to down, results for the trichromat, protanope, deuteranope and tritanope observers, when the hierarchical clustering is applied to obtain a user customized palette for the uniform color set. The left column shows the perceived colors of the 4-uniform palette, in the CIELAB space. The diamonds highlight the four colors selected. In the center the dendrogram representing the hierarchical clustering. In the left the selected palette as perceived for the observer, when four colors are needed, that is, when the dendrogram was cut at level four. 

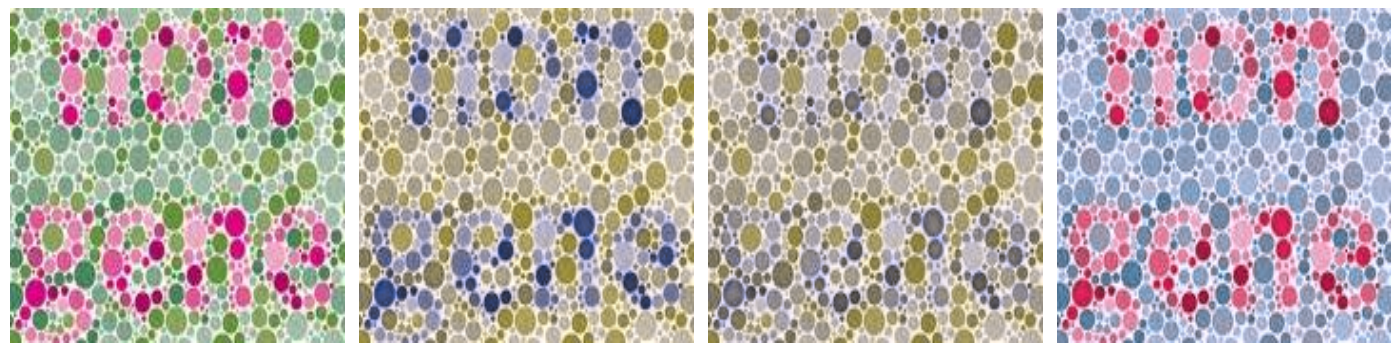

Figure 5. Ishihara style image. From left to right, renditions of the graphic representing the image perceived by a trichromat, protanope, deuteranope and tritanope. The original, i.e. the first image on the left is obtained by cropping the image provided in. ${ }^{2}$ Note that the images in this figure and the next are best viewed in electronic format on an sRGB display and printed versions may not show intended effects.

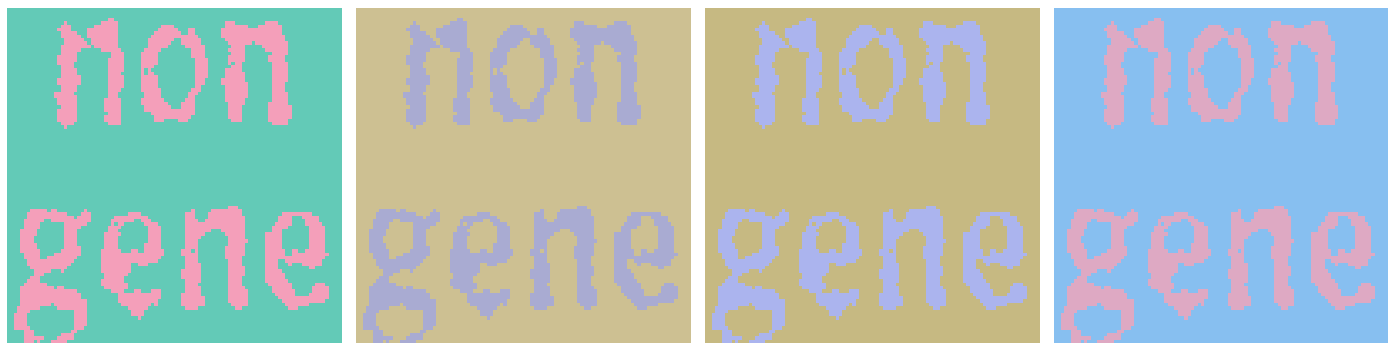

Figure 6. From top to down, results for the trichromat, protanope, deuteranope, and tritanope observers, when the hierarchical clustering is applied for the Ishihara style image.

\section{REFERENCES}

[1] "colorbrewer," http://colorbrewer2.org/, accessed November 2010.

[2] "Ishihara style image," http://www.nature.com/nature/journal/v447/n7146/full/447782a.html, accessed November 2010.

[3] "R-project," http://www.r-project.org/, accessed November 2010.

[4] "Vishek," http://www.vischeck.com/, accessed November 2010.

[5] L. D. Bergman, B. E. Rogowitz, and L. A. Treinish, "A rule-based tool for assisting colormap selection," in Proceedings of the 6th conference on Visualization. Washington, DC, USA: IEEE Computer Society, 1995, pp. 118-. [Online]. Available: http://portal.acm.org/citation.cfm?id=832271.833862

[6] H. Brettel, F. Viénot, and J. Mollon, "Computerized simulation of color appearance for dichromats," Journal of the Optical Society of America A, vol. 14, no. 10, pp. 2647-2655, 1997.

[7] C. Chen, "Top 10 unsolved information visualization problems," IEEE Computer Graphics and Applications., vol. 25, pp. 12-16, July 2005.

[8] I. C. Consortium, "International color consortium website." [Online]. Available: http://www.color.org/

[9] C. Healey, "Choosing effective colours for data visualization," in Proceedings of the 7th conference on Visualization. IEEE, 1996, pp. 263-270.

[10] J. Huang, C. Chen, T. Jen, and S. Wang, "Image recolorization for the colorblind," in IEEE International Conference on IEEE Acoustics, Speech and Signal Processing, 2009, pp. 1161-1164.

[11] J. Huang, Y. Tseng, S. Wu, and S. Wang, "Information preserving color transformation for protanopia and deuteranopia," IEEE Signal Processing Letters, vol. 14, no. 10, pp. 711-714, 2007.

[12] A. Jain and R. Dubes, Algorithms for clustering data. Prentice Hall, 1988.

[13] G. Kuhn, M. Oliveira, and L. Fernandes, "An efficient naturalness-preserving image-recoloring method for dichromats," IEEE Transactions on Visualization and Computer Graphics, pp. 1747-1754, 2008.

[14] K. Rasche, R. Geist, and J. Westall, "Detail preserving reproduction of color images for monochromats and dichromats," IEEE Computer Graphics and Applications, vol. 25, no. 3, pp. 22-30, 2005.

[15] S. Schwartz, Visual perception: A clinical orientation. McGraw-Hill Medical, 2009.

[16] G. Sharma, Ed., Digital Color Imaging Handbook. Boca Raton, FL: CRC Press, 2003. 
[17] G. Sharma and H. J. Trussell, "Digital color imaging," IEEE Trans. Image Proc., vol. 6, no. 7, pp. 901-932, Jul. 1997. [Online]. Available: http://www.ece.rochester.edu/ gsharma/papers/dciip97.pdf

[18] A. Stockman and L. Sharpe, "The spectral sensitivities of the middle-and long-wavelength-sensitive cones derived from measurements in observers of known genotype," Vision Research, vol. 40, no. 13, pp. 1711-1737, 2000 .

[19] G. Tanaka, N. Suetake, and E. Uchino, "Lightness modification of color image for protanopia and deuteranopia," Optical review, vol. 17, no. 1, pp. 14-23, 2010.

[20] H. J. Trussell and M. S. Kulkarni, "Sampling and processing of color signals," IEEE Trans. Image Proc., vol. 5, no. 4, pp. 677-681, Apr. 1996.

[21] A. Zeileis, K. Hornik, and P. Murrell, "Escaping RGBland: Selecting colors for statistical graphics," Computational Statistics \&3 Data Analysis, vol. 53, no. 9, pp. 3259-3270, 2009. 